\title{
A Pneumatic Cell Compressor
}

\author{
Noburô Kamiya and Kiyoko Kuroda* \\ Department of Cell Biology, National Institute for Basic Biology, Okazaki, \\ Aichi 444 and *Department of Biology, Faculty of Science, Osaka University, \\ Toyonaka, Osaka 560, Japan
}

\begin{abstract}
A new type of pneumatically controlled cell compressor is described. Compression without shear play was possible by the slight inward bending of a pair of coverslips which was caused by negative air pressure applied to the interior of the chamber.
\end{abstract}

There are various types of cell compressors $(1,2,3,4,5)$, but many are not precise enough or they are not convenient to handle.

The compressor which we designed and constructed is illustrated in Fig. 1 and 2. It is comparatively simple and easy to manipulate, yet it provides a degree of control sufficient for most experimental purposes. The principle of this compression chamber is to control the height of the small gap, where the cell is to be placed and flattened, by the slight inward bending of a pair of coverslips. This bending is caused by moderate negative air pressure applied to the inside of the chamber. The bending of a glass slide by a mechanical device also was used by Goldacre et al. (2) for their cell compressor.

The chamber was made of a brass frame coated with nickel, and was fitted on its top and bottom with a pair of identical coverslips $(35 \mathrm{~mm} \times 50 \mathrm{~mm}, 0.135-0.140 \mathrm{~mm}$ thick, Matsunami Glass Works, Osaka). The air space between the two coverslips was $1.85 \mathrm{~mm}$ high. Two small vents with an inner bore of $0.85 \mathrm{~mm}$ penetrated the metal frame and connected the inner air space of the chamber with the outside. A small piece of glass block $(4 \mathrm{~mm} \times 5 \mathrm{~mm}, 1.50-1.70 \mathrm{~mm}$ high $)$ was placed at the center of the bottom coverslip as a platform for the cell. Cells were placed on the platform with enough of the solution to be used so that the whole platform was immersed between the two coverslips after the top coverslip was set in position (Fig. 2a, $b_{1}$ ). The gapdistance between the upper coverslip and the platform could be adjusted to the size of the cell with a glass block of appropriate height. When the chamber was used for cell compression, one of the two vents was closed with a rubber cap. To secure airtightness, the coverslips were sealed with a small amount of vaseline and fastened by 6 screws between two metal frames with a pair of rubber gaskets inserted in between (Fig. 1).

To control the air pressure inside the chamber, one of the vents was connected by rubber tubing to an aspirator equipped with a screw-device and a water manometer. Thus, cell compression could be adjusted by remote control without touching the chamber on the microscope stage. The negative pressure we applied to the chamber was between $0-40 \mathrm{~cm}$ (water column). The relation of the negative air pressure applied to the chamber and the gap distance is shown in Fig. 3.

The gap distance was measured on the micrometer scale of the microscope with 

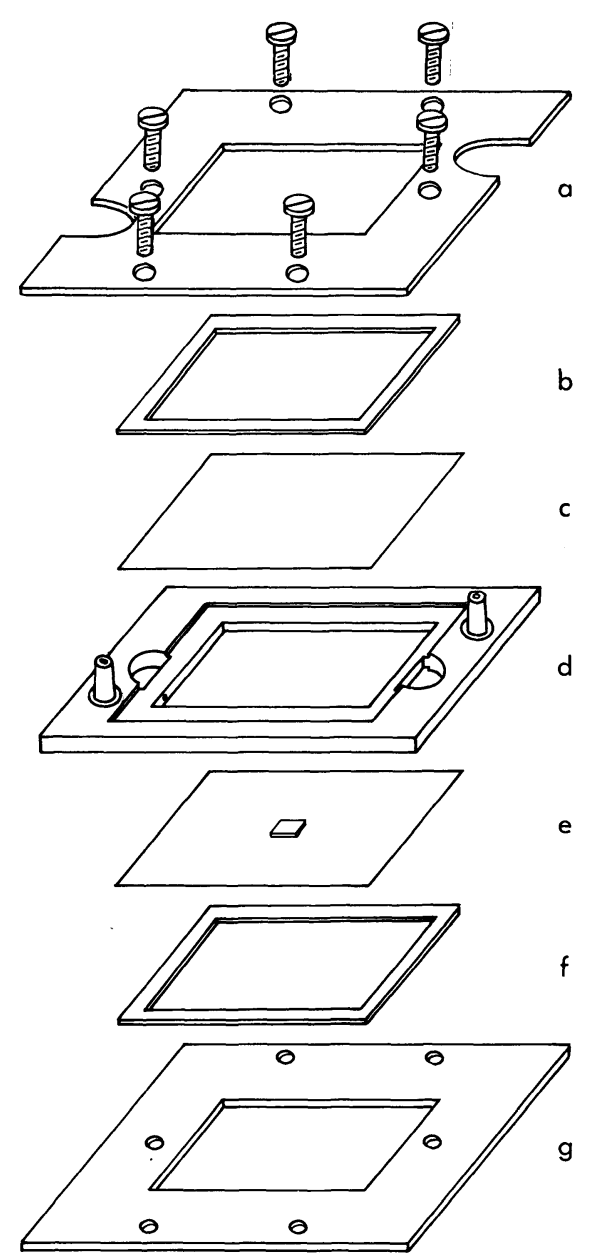

b

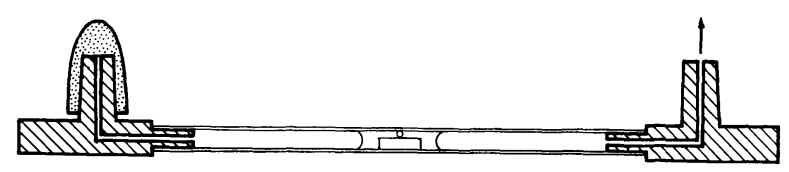

a
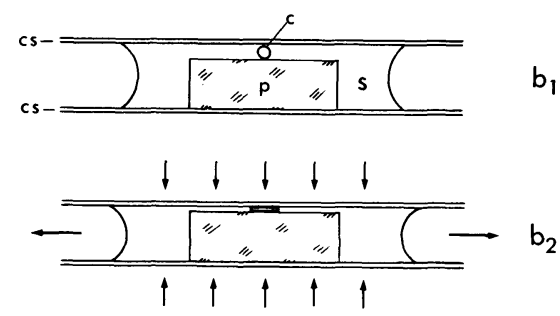

Fig. 2.

Fig. 1.

Fig. 1. Parts of the compression chamber shown in series. $d$ : main part of the chamber to be fitted with a pair of coverslips (c, e). A small glass platform that supports the samples is placed at the center of the bottom coverslip (e). b, f: silicon rubber gaskets, a, g: metal frames.

Fig. 2. Longitudinal section of the pneumatic cell compressor. a: section of the chamber. The pair of metal frames and gaskets are not indicated. For convenience, the two vents, one of which is closed with a rubber stopper, are shown. b: central part of the compressor with the platform (p) and a cell (c) immersed with a drop of solution (s) between the two coverslips (cs.) b $b_{1}$ : the zero-pressure state. $b_{2}$ : the negative pressure state that causes narrowing of the distance between the platform and the top coverslip.

index markers imprinted on the inner surface of the top coverslip and on the platform. Because there was a barely perceptible hysteresis in the gap width, the approximate width was known from the pressure applied to the chamber once a calibration curve (Fig. 3) was made. For exact quantitative work, we can check the gap distance each time with the microscope micrometer using the index markers on the coverslip and the platform, or by measuring the diameter of a flattened oil drop of known volume placed between the coverslip and the platform. 


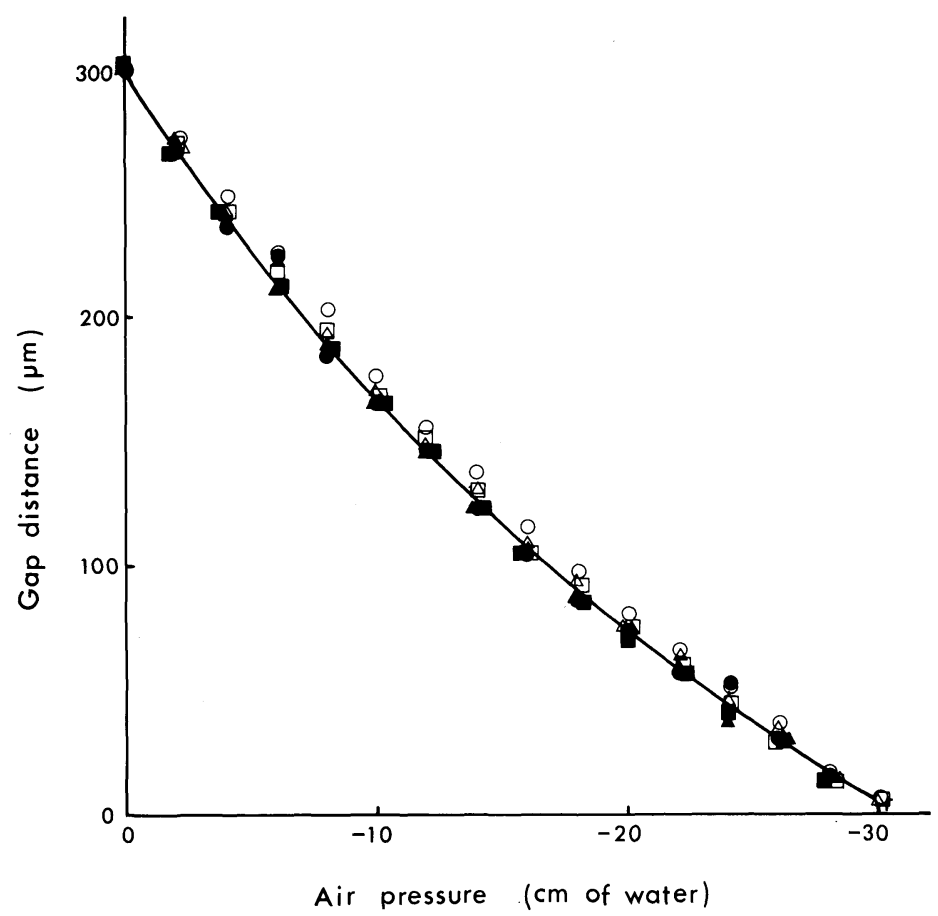

Fig. 3. Gapdistance between the coverslip and the platform surface in relation to the negative air pressure inside the chamber. This is a record of three consecutive cycle changes in the gap distance when the internal pressure was decreased and increased cyclically ( 0 to $-30 \mathrm{~cm}$ of a water column). The first, second, and third cycles are shown by circles, triangles, and squares, respectively; open symbols represent the decreasing pressure phase $(0 \rightarrow-30 \mathrm{~cm}$ of water $)$ and solid symbols the increasing pressure phase $(0 \leftarrow-30 \mathrm{~cm}$ of water $)$.

We also made another chamber which was essentially the same except that the distance between the upper and lower coverslips was narrowed to $1.00 \mathrm{~mm}$. With this chamber, it was possible to observe the specimen under Köhler illumination with an ordinary short focus condenser.

Fig. 4 shows an example of Amoeba proteus flattened in a reversible fashion. The vertical white bars at upper right in Fig. $4 \mathrm{~b}$ and $4 \mathrm{c}$ represent the gap width between the upper and lower glass surfaces in the same photographic magnification. In Fig. $4 \mathrm{a}$, where the gap distance was $300 \mu \mathrm{m}$ with no negative pressure inside, the amoeba was entirely free to move. Fig. $4 \mathrm{~b}$ (gap distance: $24 \mu \mathrm{m}$ ) shows the intermediate state between the free state and the state close to the maximal compression at which the amoeba could survive (Fig. 4c, gap distance: $14 \mu \mathrm{m}$ ). The approximate minimal thickness that the normal $A$. proteus can usually withstand is about $15 \mu \mathrm{m}$, although it depends to some extent on the speed of compression. With further flattening the cell bursts. Except when the cell burst, flattening per se did not cause appreciable harm since the process was completely reversible (Fig. 4d). An important merit in flattening the living cell is that it greatly improves visibility of cell contents. In Fig. $4 \mathrm{c}$, where the cell was compressed, the nucleus and nucleoli were much clearer than when viewed in a free cell. 

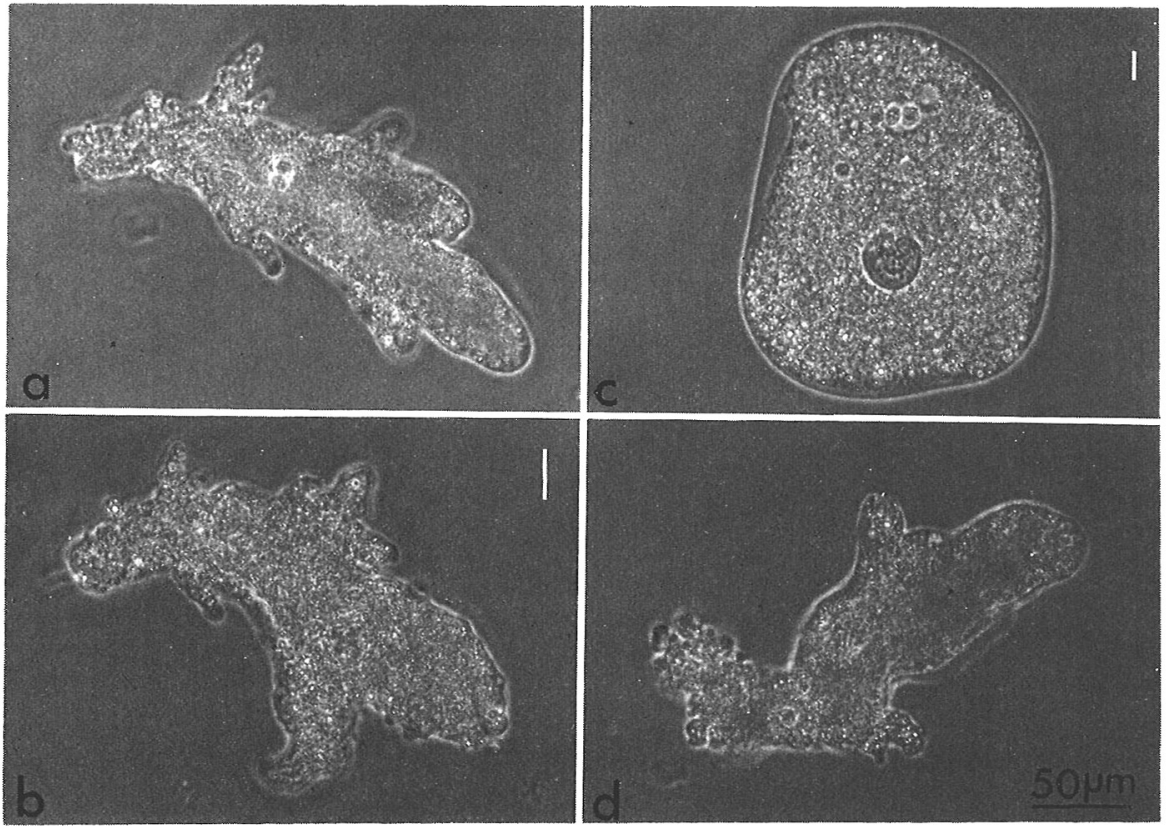

Fig. 4. Amoeba proteus flattened in a reversible fashion with the cell compressor. The gapdistance between the platform and coverslip in $4 \mathrm{a}$ and $4 \mathrm{~d}$ was $300 \mu \mathrm{m}$ (under zero pressure) with no spatial restrictions on movement. The gapdistances in $4 b$ and $4 c$ are shown by vertical white bars at upper right.
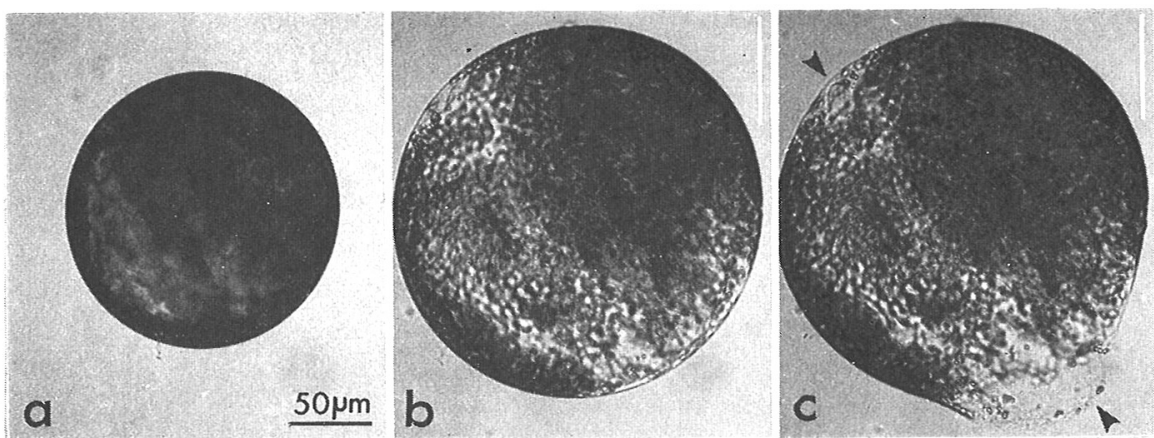

Fig 5. A protoplast from a Nitella expansa leaflet cell. Gap distances in b and c are $65 \mu \mathrm{m}$ and $63 \mu \mathrm{m}$, respectively, as shown by vertical white bars at upper right.

Fig. 5 shows another example where a large protoplast obtained from a Nitella leaflet cell was flattened. The protoplast was first completely spherical with a diameter of $167 \mu \mathrm{m}$ (Fig. 5a). Cytoplasmic streaming continued vigorously in the spherical protoplast (b) although rotational streaming was greatly distorted as a consequence of deformation from a slender cylinder to a sphere. The spherical protoplast could be flattened to a disc-like form (Fig. 5b). This flattening of the spherical protoplast is accompanied by an increase in surface area. As with the amoeba, there was a critical point at which the protoplast could no longer withstand compression. Interestingly, 
bursting always took place at two definite loci of the protoplast (Fig. 5c, arrows); the loci that originally faced the two terminal ends of the cylindrical cell in vivo.

Two examples of cell flattening with the pneumatic cell compressor we developed have been presented. This compressor also may be conveniently used for phase contrast and interference microscopy and for arresting free-swimming cells such as ciliata, swarm cells, spermatozoa, etc. by compressing them slightly, which does not cause serious harm. When necessary, we can maintain cells in the chamber under aseptic conditions in a medium with nutrients. The chamber can serve also as perfusion chamber by use of the two vents.

This work was supported in part by a grant-in-aid from the Ministry of Education, Science and Culture of Japan.

\section{REFERENCES}

1. Etzold, H. and W. HofmanN. Untersuchung lebender Mikroorganismen im abgeflachten Zustand. Microscopica Acta 77, 48-59, 1975

2. Goldacre, R.J., D.M. EASTy and E.J. Ambrose. A cell compressor for the measurement of mass and concentration by interference microscopy. Nature 180, 1487-1488, 1957

3. HeunerT, H.H. Erfahrungen mit einer neuen Kammer zur Lebendbeobachtung beweglicher Mikroorganismen. Research Film 5, 642-649, 1966

4. Heunert, H.H. Microtechnique for the observation of living microorganisms. Zeiss Inform. 20, 37-68, 1973

5. INOUÉ, S. A method to modify at will the distance between coverslip and glass slide. Botany and Zoology 11, 669, 1943 (in Japanese)

6. Kuroda, K. and N. KamiYa. Surgical operations in giant plant cells and their applications. Seitai no Kagaku 26, 59-66, 1975 (in Japanese) 